\title{
Pneumocystis Pneumonia in Systemic Lupus Erythomatosus: A Case Report
}

\section{Sultana $\mathrm{T}^{1 *}$, Allam Choudhury $\mathrm{A}^{2}$, Rahman $\mathbf{Q}^{3}$, and Nashimuddin}

\section{Ahmed AN 4}

1Professor, Department of Clinical Pathology, BSMMU, Bangladesh

2Department of Otolaryngology- Head \& Neck Surgery, BSMMU, Bangladesh

${ }^{3}$ Professor, Department of Clinical Pathology, BSMMU, Bangladesh

${ }^{4}$ Professor and Chairman, Department of Clinical Pathology, BSMMU, Bangladesh

\section{Case Report \\ Volume 1 Issue 1}

Received Date: May 13, 2017

Published Date: May 25, 2017

DOI: $10.23880 /$ cprj-16000103

*Corresponding author: Tuhin Sultana, Department of Clinical Pathology, BSMMU, Bangladesh, E-mail: drtsultana@gmail.com

\section{Abstract}

Patients with systemic lupus eythematosus (SLE) have increased susceptibility to infection by Pneumocystis jerovecii but this condition has rarely been reported in Bangladesh. Pneumonias due to Pneumocystis jerovecii commonly occur in immunocompromised hosts. Although it is a treatable infection, it is associated with high motility. Patient with systemic lupus erythomatosus increased susceptibility to infection by Pneumocystis jerovecii. Here we describe a patient with SLE who developed Pneumocystis pneumonia (PCP). A 37-years old female was a known case of SLE for 12 years admitted in BSMMU with the complaints of fever \& cough for 3 months and breathlessness for 1 month. The patient was treated with corticosteroids and cyclosporine within 2 months before presentation. Diagnosis was established based on the findings of induced sputum by Giemsa staining. This case demonstrated that PCP should be included in the differential diagnosis of patients of SLE presenting with pneumonic process.

Keywords: Pneumocystis jerovecii; Pneumocystis pneumonia (PCP); Systemic lupus erythomatosus (SLE)

\section{Introduction}

The occurrence of pneumocystis pneumonia in patient with collagen disease on immunosuppressive therapy is not uncommon. But only a few case reports are available about this infection in active untreated collagen disorders. The diagnosis of PCP is done either by induced or spontaneous sputum analysis, or by carrying out a bronchoalveolar lavage and transbronchial lung biopsy. Recently we encountered a case of PCP in SLE patient where definitive diagnosis based on the demonstration of Pneumocystis jerovecii obtained from induced sputum

\section{Case Summary}

A 37 years old female, non diabetic mother of one child hailing from Fenny was a known case of SLE for 12 years admitted in BSMMU with the complained of fever \& cough for 3 months and breathlessness for 1month .On examination she was mild anaemic, oral ulcer present in her tongue \& hard palate. Her BP $80 / 60 \mathrm{mmHg}$, RR 42 
breathes/min, chest expansibility reduced to $3 \mathrm{~cm}$, coarse cripitation present throughout the chest which alter with coughing.

Regarding drug history she was on long term immunosupressive therapy, she was treated with methotrixate 20mg for 10years (2003-2013), azathioprine 75mg for 2 years (Oct, 2013-Jan, 2015), cyclosporin 200mg for 3 months (Jan, 2015-March, 2015) \& with prednisolone $1 \mathrm{mg} / \mathrm{kg}$ body weight to gradual tapering dose (Oct, 2013-Nov, 2014) \& currently she was being treated with prednisolone scince January 2015.

She was evaluated for the cause of breathlessness. Urine \& blood culture was sterile. Sputum examination did not show acid fast bacilli. Chest X-ray revealed bilateral diffuse infiltrate, more in perihilar regions. CT scan of chest showed bilateral diffuse infiltrate, more in perihilar regions, nodular densities. Echocardiography showed mild pericardial effusion, EF 67\%, pulmonary arterial pressure $45 \mathrm{mmHg}$. ECG showed normal findings. Laboratory report reveals $\mathrm{Hb}-11.2 \mathrm{~g} / \mathrm{dl}, \mathrm{WBC}-9500 /$ cumm with 93\% neutrophil, $06 \%$ lymphocytes, and $1 \%$ monocytes, ESR-120mm in 1st hour and platelet count $3,00,000$, Alanine aminotransferase $15 \mathrm{U} / \mathrm{L}$, S.ceatinine $1.06 \mathrm{mg} / \mathrm{dl}$. First sputum for pneumocystis jerovecii showed negative but induced sputum by $3 \% \mathrm{NaCl}$ showed pneumocystis jerovecii by Giemsa stain done at Clinical Pathology Department of BSMMU (Figures 1A and 1B).
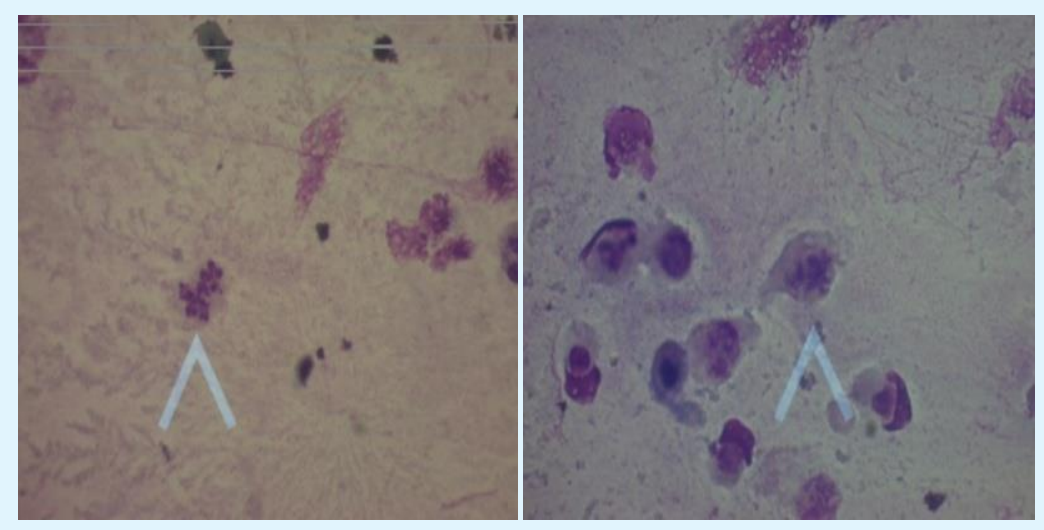

Figure 1A and 1B: Sputum smear stained with Giemsa stain shows Pneumocystis jerovecii (oil immersion).

\section{Discussion}

Pulmonary manifestations of connective tissue diseases are a diagnostic challenge to the clinicians. It could be disease related due to immune mediate insult, vasculities, pulmonary hemorrhage, and pulmonary hypertension or caused by infection. Opportunistic infections with organism like pneumocystis jerovecii frequently complicate immunosuppressive status.

The mechanism of immune suppression in patients with SLE who have PCP is usually multi-factorial2, and may be related to underlying diseases, cytotoxic therapies, or malnutrition. However, the development of PCP in most patients with SLE is associated with daily administration of corticosteroids and with the development of lymphopenia3. Corticosteroids cause immunosuppression mainly by sequestration of CD4+Tlymphocytes in the reticuloendothelial system and by inhibiting the transcription of cytokines4-5. Corticosteroid therapy is a rare but possible independent predisposition to Pneumocystis jirovecii infection 6-7.
Prolonged corticosteroid therapy is characterized by a significant immunological dysfunction.

In this case patient was treated by cyclosporin with prednisolone and she was also lymphopenic, may decrease CD4 count8. This impairs cellular immunity and predispose to opportunistic infection like pneumocystis jerovecii. The diagnosis of PCP was made by sputum analysis in our case. The sensitivity of the sputum analysis is $50-60 \%$, though less sensitive 9 , sputum examination is highly specific for organism 10 .

\section{Conclusion}

Pneumocystis pneumonia is a fatal disease. Pneumocystis pneumonia occurs mostly immunocompromised patient. The patient response well to the anti pneumocystis treatment. The clinicians should be aware that, at some point of time immunosuppressed patients can present with concurrent infections with Pneumocystis jirovecii. 


\section{References}

1. Barshes NR, Goodpastor SE, Goss JA (2004) Pharmacologic immunosuppression. Front Biosci 9: 411-420.

2. Farr RW (1992) Pneumocystis carinii pneumonia due to corticosteroids. South Med J 85(1): 52-53.

3. Toledo AC Jr, de Castro MR (2001) Pneumocystis carinii pneumonia, pulmonary tuberculosis and visceral leishmaniasis in an adult HIV negative patient. Braz J Infect Dis 5(3): 154-157.

4. Roux N, Flipo RM, Cortet B, Lafitte JJ, Tonnel AB, et al. (1996) Pneumocystis carinii pneumonia in rheumatoid arthritis patients treated with methotrexate. A report of two cases. Rev Rhum Engl Ed 63(6): 453-456.

5. Slivka A, Wen PY, Shea WM, Loeffler JS (1993) Pneumocystis carinii pneumonia during steroid taper in patients with primary brain tumors. Am J Med 94(2): 216-219.
6. Sepkowitz KA, Brown AE, Telzak EE, Gottlieb S, Armstrong D (1992) Pneumocystis carinii pneumonia among patients without AIDS at a cancer hospital. JAMA 267(6): 832-837.

7. Arend SM, Kroon FP, van't Wout JW (1995) Pneumocystis carinii pneumonia in patients without AIDS, 1980 through 1993. An analysis of 78 cases. Arch Intern Med 155(22): 2436-2441.

8. Filoche P, Adoun M, Caron F, Godet C, Robert R, et al. (2006) Co-infection with Mycobacterium tuberculosis and Pneumocystis jirovecii in a patient without HIV infection. Rev Mal Respir 23: 83-87.

9. Koffi N, Ngom A, Aka Danguy E (1997) Association of pneumocystosis and pulmonary tuberculosis in a HIV negative patient. Rev Mal Respir 14(5): 399-400.

10. Takamura N, Kimura A, Hamamoto $Y$ (1975) An autopsy case of lymphosarcoma associated with Pneumocystis carinii, cytomegalovirus infection, and miliary tuberculosis. Bull Osaka Med Sch 21: 108-118. 